\title{
VIGILÂNCIA DO DESENVOLVIMENTO INFANTIL: ANÁLISE DOS FATORES DE RISCO PARA CRIANÇAS MENORES DE DOIS ANOS
}

\author{
Hyana Pereira Dias ${ }^{1}$ Ana Gabriela Alves de Lima1 Julyana Karollyny Barros \\ Faustino ${ }^{1}$ Francisco de Assis Coutinho Pontes Júnior ${ }^{2}$ Moema Teixeira Maia ${ }^{3}$ \\ Isolda Maria Barros Torquato ${ }^{3}$ \\ ${ }^{1}$ Enfermeira, Unidade Acadêmica de Enfermagem, Universidade Federal de Campina Grande, \\ Cuité-PB, Brasil. \\ 2Fisioterapeuta, Hospital Universitário Lauro Wanderley, João Pessoa, PB, Brasil. \\ ${ }^{3}$ Docente, Departamento de Fisioterapia, Universidade Federal da Paraíba, João Pessoa, PB, \\ Brasil. \\ E-mail para correspondência: isoldaufcg@gmail.com
}

\begin{abstract}
Resumo
O desenvolvimento infantil é resultado da interação entre os fenômenos biológicos e ambientais. Realizar a vigilância do desenvolvimento é essencial para que haja a identificação de fatores de risco. Objetivou-se analisar os fatores de risco para o desenvolvimento infantil em crianças menores de dois anos. Participaram 50 mães de crianças menores de 24 meses, usuárias de Centros de Referência em Educação Infantil de Cuité. Aplicou-se um formulário estruturado, contendo 42 questões sobre os fatores de riscos para o desenvolvimento infantil. A análise dos dados ocorreu por estatística descritiva. O trabalho foi aprovado por Comitê de Ética em Pesquisa sob parecer 1.828.821. A maioria das mães apresenta idade entre $19-29$ anos $(56,0 \%)$, solteira $(52,0 \%), 15$ ou mais anos de estudo $(28,0 \%)$, renda familiar menor que um salário mínimo $(64,0 \%)$. Cerca de $18 \%$ referiram depressão no pós parto e $42 \%$ revelaram insatisfação com a relação conjugal. A maioria das crianças possui idades entre 1 a 2 anos (84,0\%), sendo do sexo feminino $(52,0 \%)$. Cerca de $16,0 \%$ foram prematuras e apresentaram icterícia. Constatou-se a presença de fatores de risco importantes, sendo fundamental a realização de intervenções educativas pautadas na promoção do desenvolvimento infantil a fim de prevenir possíveis alterações na criança.
\end{abstract}

Palavras-chave: Vigilância, Fatores de risco, Desenvolvimento infantil, Crianças.

\begin{abstract}
Child development is the result of the interaction between biological and environmental phenomena. Performing development surveillance is essential for identifying risk factors. The objective was to analyze the risk factors for child development in children under two years. Participants were 50 mothers of children under 24 months, who used Cuité Reference Centers for Early Childhood Education. A structured form containing 42 questions on risk factors for child development was applied. Data analysis was performed using descriptive statistics. The study was approved by the Research Ethics Committee under opinion 1.828.821. Most mothers are between $19-29$ years old $(56.0 \%)$, single $(52.0 \%), 15$ or more years of schooling $(28.0 \%)$, family income less than one minimum wage (64.0\%). \%). About $18 \%$ reported postpartum depression and $42 \%$ revealed dissatisfaction with their marital relationship. Most children are between 1 and 2 years old $(84.0 \%)$, being female $(52.0 \%)$. About $16.0 \%$ were premature and
\end{abstract}


had jaundice. It was found the presence of important risk factors, and it is essential to carry out educational interventions based on the promotion of child development in order to prevent possible changes in the child.

Keywords: Surveillance, Risk factors, Child development, Children

\section{Introdução}

O desenvolvimento infantil abrange o crescer físico, a maturação neurológica e a constituição de habilidades do comportamento (DAELMANS et al., 2015). Segundo estudo (SILVA; GONTIJO, 2015), está relacionado às capacidades motoras e psicocognitivas, de modo sistemático e sequencial.

Trata-se de um processo resultante da interação entre os fenômenos de crescimento e aprendizagem, onde ocorrem mudanças que podem ser notadas nas dimensões física, intelectual, emocional e social (CAMINHA et al., 2017). Contudo, durante o desenvolvimento infantil fatores de risco, como os genéticos, biológicos e ambientais, podem comprometer o seguimento sequencial esperado (SOUZA; VERÍSSIMO, 2015).

Avalia-se que, uma em cada oito crianças apresenta alterações do desenvolvimento que interferem em sua qualidade de vida e inclusão na sociedade. Desse modo, a identificação desses fatores, assim como a intervenção precoce são aspectos decisivos para o prognóstico de crianças em risco para o desenvolvimento (SOUZA et al., 2019).

Nesse contexto, destaca-se a importância das ações de vigilância em saúde da criança, abrangendo programas diferenciados, especificamente a vigilância do desenvolvimento infantil no contexto da Atenção Integrada as Doenças Prevalentes na Infância (AIDPI), que compreende todas as atividades relacionadas à promoção do desenvolvimento e detecção de desvios (REICHERT et al, 2015).

Como instrumento de avaliação dos fatores de risco e do desenvolvimento infantil, a AIDPI colocou à disposição das famílias um conjunto de intervenções que permitem aos profissionais de saúde aplicar medidas de avaliação e promoção da saúde integral proporcionando conhecimento e prática para os pais, no cuidado dos filhos (TORQUATO et al., 2019). 
O acompanhamento do desenvolvimento deve ser feito no contexto da atenção primária à saúde, e necessita ter o apoio da família, comunidade e profissionais da saúde. Nesse sentido, cabe a estes últimos, ofertarem às orientações importantes no cuidado às crianças, intervindo quando preciso (GAívA et al., 2018).

É mister ressaltar que identificar os fatores de risco envolvidos, além de analisar o conhecimento dos pais frente ao desenvolvimento infantil é essencial, para que as orientações adequadas possam ser ofertadas, conforme as necessidades apresentadas. Desta forma, elas também terão mais possibilidades de cuidar e estimular seus filhos de maneira correta e eficaz (EYKEN et al., 2015).

Deste modo, considerando os aspectos acima citados e a relevância da temática para aquisição de evidências buscou-se responder ao seguinte questionamento: Quais os fatores de risco para o desenvolvimento infantil em crianças menores de dois anos? Com isso, objetivou-se, nesse estudo, analisar os fatores de risco para o desenvolvimento infantil em crianças menores de dois anos.

\section{Metodologia}

Trata-se estudo transversal, exploratório, descritivo com abordagem quantitativa. O estudo foi desenvolvido, outubro de 2016 a janeiro de 2017, em Centros de Referência Infantil (CREI) do município de Cuité - PB, localizadas na zona urbana da região do Curimataú Ocidental Paraibano. A escolha do referido cenário é justificável devido as instituições assistirem em tempo integral crianças com faixas etárias variadas e por constituírem-se espaços fundamentais para a realização de vigilância da saúde da criança.

Participaram da pesquisa 50 mães de crianças menores de dois anos matriculadas e que estivessem frequentando o CREI durante o período de coleta de dados. Foram excluídas, as mães com distúrbios mental e de comunicação. A justificativa para a escolha desta faixa de idade baseia-se na recomendação do Ministério da Saúde (BRASIL, 2017), o qual preconiza essa 
faixa etária como um período crítico para o desenvolvimento neuropsicomotor da criança.

A coleta de informações ocorreu por meio do uso de um formulário elaborado a partir do Manual de Vigilância do Desenvolvimento da AIDPI (BRASIL, 2017). As 42 questões objetivas, sobre os fatores de risco relacionados ao desenvolvimento infantil, envolveram dados maternos: sociodemográficos (Escolaridade materna, trabalho remunerado, renda familiar, moradia com saneamento básico, tipo de moradia e número de cômodo no domicílio), reprodutivos (idade materna, intervalo interpartal, realização do pré-natal, primeira consulta do pré-natal, intercorrências, consumo de álcool, tabaco e drogas ilícitas durante a gestação), saúde materna (problemas de saúde e utilização de medicamentos), satisfação conjugal (satisfação com o relacionamento conjugal, rejeição da gravidez e presença de brigas conjugais cotidianamente), assim como informações sobre rede de apoio (recebe apoio do pai, familiares e de outras redes de apoio para o cuidado à criança); e da criança sobre condições do nascimento (idade, sexo, peso ao nascer, idade gestacional, altura e peso atual, índice de massa corporal e morbidade neonatal); saúde da criança (hospitalização, vacinas, consulta de puericultura no primeiro ano de vida, estado nutricional, amamentação); e estrutura familiar (pais residem juntos e número de irmãos).

As entrevistas foram realizadas nos turnos matutino (07:00 às 12:00 horas) e vespertino (13:00 às 17:00 horas), conforme os dias e horários estabelecidos pelas coordenadoras dos CREl's envolvidos.

Os dados foram analisados descritivamente sob a forma de tabelas, sendo discutidos a luz da literatura pertinente. O estudo foi aprovado pelo Comitê de Ética em Pesquisa sob CAAE n 61529916.1 .0000 .5182 e parecer no 1.828.821, conforme exigências estabelecidas pela Resolução 466/2012 do Conselho Nacional de Saúde, que norteia a prática de pesquisa com seres humanos.

\section{Resultados e Discussão}


Conforme dados obtidos das 50 participantes (Tabela 1), a maioria apresentou idade entre 19 e 29 anos (56,0\%). Apesar de não retratar os dados da presente pesquisa, os extremos idade materna, segundo estudo (SILVA et al., 2015) é uma variável que influencia no desenvolvimento infantil, visto que, àquelas que se encontram nessa condição possuem mais chances para intercorrências e desenvolvimento de doenças que resultem risco para a díade mãe-filho.

A maioria das mães era solteira $(52,0 \%)$, dedicando-se aos cuidados do lar e do filho. Conforme pesquisa, a ausência da figura paterna pode colaborar para uma gestação mais estressante devido à sobrecarga de tarefas para a mulher, especialmente quando não há apoio familiar. Nesse sentido, mães solteiras tem maior probabilidade em ter recém-nascidos prematuros, sendo a prematuridade, um importante fator de risco para o desenvolvimento infantil (NAZARETH, SANTOS, GOLÇALVES; SOUZA, 2014).

A maioria das mães apresentou nível de escolaridade variando entre 15 ou mais anos de estudo (28,0\%). Para os autores, mães com maior nível de escolaridade apresentam respostas mais elaboradas e melhores informações acerca do conhecimento sobre desenvolvimento de seus filhos contrariamente àquelas com baixa escolarização, que, muitas vezes, não possuem acesso a informação e tomada de decisão para promover o desenvolvimento da criança (MATOS; CRUZ; CAVALCANTE, 2017).

Quanto ao trabalho remunerado, os resultados, semelhantes ao estudo desenvolvido em São Paulo (CID, 2015), evidenciaram que 68,0\% não trabalha fora do domicílio, o que contribui para uma menor concentração de renda familiar. Apesar de ser uma condição que possivelmente aproxima a mãe dos cuidados do filho, conforme o autor, a renda baixa pode dificultar a oferta dos insumos adequados à criança, a exemplo de alimentação e acesso a saúde e lazer, essenciais para o desenvolvimento saudável.

Segundo pesquisa (GERZSON et al., 2016), crianças que vivem em condições econômicas desfavoráveis nos primeiros anos de vida podem apresentar dificuldades em todas as áreas do desenvolvimento, devido a família não apresentar condições financeiras que ofereça recursos para estimular os diferentes sentidos. Este fator de risco, é perceptível na pesquisa 
atual, considerando que $64,0 \%$ das entrevistadas apresentaram renda familiar menor que um salário mínimo.

O fato das moradias não apresentarem saneamento básico evidenciam uma exposição maior para as crianças quanto às doenças parasitárias e hospitalizações, com consequente risco para o desenvolvimento global. Conforme estudo (PAIVA; SOUZA, 2018), as internações por doenças veiculadas pela água estão associadas também a baixa escolaridade e cobertura por serviços de atenção básica.

Cerca de $44,0 \%$ das famílias vivem em casas com 4 ou 5 cômodos. Conforme estudo (NAZARETH, SANTOS, GOLÇALVES; SOUZA, 2014), moradias com números de cômodos reduzidos possibilitará menos espaços disponíveis para a criança se locomover, realizar diversas atividades, explorar e interagir com o meio, podendo se tornar um aspecto de risco para o desenvolvimento.

Tabela 1: Características sociodemográficas de mães de crianças usuárias de CREl's do município de Cuité. Brasil. Paraíba. 2017.

\begin{tabular}{|l|c|c|}
\hline \multicolumn{1}{|c|}{ Variáveis } & N & $\%$ \\
\hline Idade & 7 & 14,0 \\
\hline$<19$ anos & 28 & 56,0 \\
\hline $19-29$ anos & 14 & 28,0 \\
\hline $30-40$ anos & 1 & 2,0 \\
\hline$>40$ anos & 26 & 52,0 \\
\hline Estado civil & 23 & 46,0 \\
\hline Solteira & 1 & 2,0 \\
\hline Casada & & 8,0 \\
\hline Desquitada & 4 & 22,0 \\
\hline Escolaridade & 11 & 12,0 \\
\hline Sem instrução & 6 & 16,0 \\
\hline 1 a 3 anos de estudos & 8 & 14,0 \\
\hline 4 a 7 anos de estudos & 7 & 28,0 \\
\hline 8 a 10 anos de estudos & 14 & 32,0 \\
\hline 11 a 14 mais anos de estudos & & 68,0 \\
\hline 15 ou mais anos de estudos & 16 & 64,0 \\
\hline Trabalho remunerado & 34 & 28,0 \\
\hline Sim & & 6,0 \\
\hline Não & 32 & 14 \\
\hline Renda Familiar & 3 & \\
\hline Menor que um salário mínimo & & \\
\hline Um salário mínimo & & \\
\hline Dois a três salários mínimos & & \\
\hline
\end{tabular}




\begin{tabular}{|l|c|c|}
\hline Maior que um salário mínimo & 1 & 2,0 \\
\hline Moradia com saneamento básico & & 0,00 \\
\hline Sim & 0 & 100,0 \\
\hline Não & 50 & \\
\hline Número de cômodos & 22 & 44,0 \\
\hline 2 a 3 & 22 & 44,0 \\
\hline 4 a 5 & 6 & 12,0 \\
\hline Mais que 5 & $\mathbf{5 0}$ & $\mathbf{1 0 0 , 0}$ \\
\hline Total &
\end{tabular}

Fonte: Dados da pesquisa, Cuité (2017).

O número elevado de filhos, não prevaleceu na amostra pesquisada. Conforme pesquisas (MATOS; CRUZ; CAVALCANTE, 2017; NAZARETH, SANTOS, GOLÇALVES; SOUZA, 2014), uma família com número elevado de filhos pode comprometer a oferta de cuidado e atenção adequada por parte dos pais, repercutindo negativamente no desenvolvimento da criança. Atrelado a isso, esse aspecto pode se tornar ainda mais preocupante quando a prática de cuidado é realizada por um cuidador apenas.

No que se refere ao intervalo interpartal $54,0 \%$ das mulheres tiveram filhos com intervalo menor que 12 meses, tendo estas mulheres à responsabilidade de cuidar ao mesmo tempo de dois ou mais filhos de idade aproximada, o que pode dificultar não apenas os cuidados específicos para cada um, mas também à disponibilidade de tempo para prover a estimulação das crianças, conforme as suas necessidades.

Ademais, é importante mencionar que há possibilidade do surgimento de intercorrências relacionadas à saúde da mulher como efeitos adversos na gestação ou no parto, devido a um intervalo de tempo menor entre uma gravidez e outra.

Outro fator de risco para o desenvolvimento infantil remete-se ao número insuficiente de consultas pré-natais como fator de risco (ROSA; SILVEIRA; COSTA, 2014). Neste estudo, porém, não se observou essa exposição, pois $92,0 \%$ das mulheres externaram ter realizado pelo menos 6 consultas prénatais, conforme preconiza o Ministério da saúde (2012), sendo a primeira consulta realizada antes dos 3 meses de gestação. Conforme estudo (CORRÊA et al., 2014), as consultas pré-natais no início da gestação são de fundamental importância para a mulher e o feto, pois poderá evitar 
complicações como óbitos, patologias e malformações que possam comprometer o desenvolvimento infantil futuro.

O consumo de álcool e tabaco, apesar de pouco expressivo neste estudo (22,0\%) também podem afetar o desenvolvimento da criança, considerando que ambos apresentam efeitos deletérios ao desenvolvimento fetal no pré e pós-natal. Ademais, estão associados a várias causas de doenças e morte perinatal, sendo assim, as gestantes estão menos sujeitas a este fator, quando não há exposição a essas substâncias (COSTA et al., 2014).

Com relação a essas drogas pesquisa (RIBEIRO; PEDROSA; PADOVANI, 2014) evidencia o fumo e o álcool como os maiores agentes externos causadores de deformidades fetais. $O$ consumo durante o período da gravidez facilita o parto prematuro e de baixo peso. Além disso, pode causar ao feto problemas de memória e aprendizado, aborto, má formação cardíaca e de outros órgãos.

A automedicação e o uso de medicamentos durante a gestação de forma irracional constituem uma conduta de alto risco, podendo ser considerado um sério problema para o desenvolvimento da criança, uma vez que nenhum medicamento é livre de toxicidade à mãe ou ao feto, especialmente no primeiro trimestre quando ocorre o processo de formação neuronal do feto (ANDRADE et al., 2014). Um aspecto positivo obtido na presente pesquisa é que $56,0 \%$ das gestantes não estiveram expostas a este fator de risco e as que consumiram afirmaram ter realizado sob orientação médica.

O estado emocional materno também é considerado um importante fator de risco para o desenvolvimento infantil. A depressão e o estresse emocional em excesso durante o período gestacional e pós-natal pode ser transferido para o feto o que pode causar aumento do movimento fetal e, após o nascimento, choro excessivo, irritabilidade, além de problemas digestórios na criança (RIBEIRO; PEDROSA; PADOVANI, 2014).

Apesar da maioria das mulheres negarem este tipo de alteração durante a gestação ou no pós-parto $(82,0 \%)$ observa-se que um percentual referiu alterações emocionais, condição que pode interferir nas relações afetivas entre 
a díade mãe-filho e consequentemente no desenvolvimento global da criança (Tabela 2).

Tabela 2: Características biológicas e obstétricas de mães de crianças usuárias de CREl's do município de Cuité. Brasil. Paraíba. 2017.

\begin{tabular}{|c|c|c|}
\hline Variáveis & $\mathbf{n}$ & $\%$ \\
\hline \multicolumn{3}{|l|}{ Números de filhos } \\
\hline 1 & 15 & 30,0 \\
\hline 2 & 20 & 40,0 \\
\hline 3 & 6 & 12,0 \\
\hline 4 & 5 & 10,0 \\
\hline 5 ou mais & 4 & 8,0 \\
\hline \multicolumn{3}{|l|}{ Intervalo Interpartal } \\
\hline$<12$ meses & 27 & 54,0 \\
\hline $12-18$ meses & 13 & 26,0 \\
\hline$\geq 19$ meses & 10 & 20,0 \\
\hline \multicolumn{3}{|l|}{ Tipo de parto } \\
\hline Normal & 20 & 40,0 \\
\hline Cirúrgico & 30 & 60,0 \\
\hline \multicolumn{3}{|l|}{ Local do parto } \\
\hline Domicílio & 1 & 2,0 \\
\hline Hospital & 49 & 98,0 \\
\hline \multicolumn{3}{|c|}{ Realização de consultas pré-natais de rotina } \\
\hline Sim & 46 & 92,0 \\
\hline Não & 4 & 8,0 \\
\hline \multicolumn{3}{|c|}{ Período da primeira consulta do pré-natal } \\
\hline$<3$ meses & 32 & 64,0 \\
\hline$\geq 3$ meses & 18 & 36,0 \\
\hline \multicolumn{3}{|c|}{ Fez uso de álcool durante a gestação } \\
\hline Sim & 7 & 14,0 \\
\hline Não & 43 & 86,0 \\
\hline \multicolumn{3}{|c|}{ Realizou uso de tabaco durante a gestação } \\
\hline $\operatorname{Sim}$ & 4 & 8,0 \\
\hline Não & 46 & 92,0 \\
\hline \multicolumn{3}{|c|}{ Fez uso de medicamentos na gestação } \\
\hline $\operatorname{Sim}$ & 22 & 44,0 \\
\hline Não & 28 & 56,0 \\
\hline \multicolumn{3}{|c|}{ Apresentou depressão na gestação ou pós-parto } \\
\hline $\operatorname{Sim}$ & 9 & 18,0 \\
\hline Não & 41 & 82,0 \\
\hline Total & 50 & 100,0 \\
\hline
\end{tabular}

Fonte: Dados da pesquisa, Cuité (2017).

Em relação ao grau de parentesco entre os pais das crianças (Tabela 3), cerca de $98 \%$ das mulheres respondeu não apresentaram consanguinidade com o pai da criança, o que diminui as chances do nascimento de crianças 
portadoras de doenças hereditárias por fatores genéticos. Para Kim, Albano; Bertola (2019), a consanguinidade entre os pais é um aspecto que favorece o aparecimento de doenças autossômicas recessivas que podem levar ao atraso do desenvolvimento neuropsicomotor. Sendo assim, a coleta do histórico familiar é necessária, principalmente em crianças que nascem com malformação, para auxiliar e esclarecer se os problemas apresentados são de origem genética.

No presente estudo, a maioria das mães referiram ter desejado a gravidez $(66,0 \%)$. É importante ressaltar que esse planejamento pode repercutir positivamente nos cuidados pré-natais, considerando que estes são mais intensos entre àquelas que planejam uma gravidez, fazendo-as frequentarem os serviços de saúde mais cedo e de forma assídua, além de estarem em harmonia com a gestação. Um filho indesejado pode ser ignorado, sendo, muitas vezes negligenciado em relação aos cuidados essenciais e na evolução do desenvolvimento normal.

O conflito conjugal também fora analisado nesta amostra. Conforme Melo e Miccione (2014), a presença de conflitos e separação dos pais pode prejudicar o desenvolvimento e o bem-estar dos filhos, considerando-se que, de alguma forma, as crianças estão expostas às brigas entre os pais. Quando os filhos testemunham os conflitos isso pode gerar comprometimento psicológico, provocando repercussões para o desenvolvimento infantil e nas relações familiares.

Tabela 3: Informações maternas sobre grau de parentesco e presença de conflitos conjugais entre os pais de crianças usuárias de CREl's do município de Cuité. Brasil. Paraíba. 2017.

\begin{tabular}{lcc}
\multicolumn{1}{c}{ Variáveis } & n & $\%$ \\
\hline Grau de parentesco entre os pais & 1 & 2,0 \\
Sim & 49 & 98,0 \\
Não & 33 & 66,0 \\
A gravidez foi desejada & 17 & 34,0 \\
Sim & & \\
Não & 29 & 58,0 \\
Apresenta satisfação com o relacionamento conjugal & 21 & 42,0 \\
Sim & 16 & 32,0 \\
Não & & \\
Apresenta conflitos conjugais & & \\
Sim
\end{tabular}




\begin{tabular}{lcc}
\hline Não & 34 & 68,0 \\
Total & $\mathbf{5 0}$ & $\mathbf{1 0 0 , 0}$ \\
\hline
\end{tabular}

Conforme dados apresentados na Tabela 4, a maioria das crianças apresentava idade entre 12 e 24 meses (84\%), sexo feminino (52,0\%) e com idade gestacional A Termo, ou seja, de 37 semanas. Esse resultado mostra que a predominância das crianças apresentou idade gestacional dentro dos parâmetros esperados de normalidade quanto a maturidade funcional e estrutural dos órgãos e tecidos. Crianças com nascimento inferior ao supracitado estão mais susceptíveis a desvios no desenvolvimento, em termos qualitativos quando comparada a crianças nascidas no período correto (ZELKOWITZ, 2017).

O baixo peso ao nascimento é um dos principais preditores de morbidade e mortalidade neonatal e perinatal. Constitui-se como um importante indicador da saúde da população infantil e fator de risco significativo para alterações no desenvolvimento motor e futura evolução neuropsicomotora da criança (FUENTEFRIA; SILVEIRA; PROCIANOY, 2017).

Isso ocorre, visto que, a criança pré-termo parece exibir um padrão de desenvolvimento lento, congruente com a ideia de que a prematuridade leva à dificuldade para entender ou processar as informações sensoriais para o controle do movimento (MOREIRA; MAGALHÃES; ALVES, 2014).

A icterícia, outro fator de risco desenvolvimental, presente em 16,0\% da amostra, é um dos sinais clínicos mais observados no período neonatal, sendo causado pelo acúmulo de bilirrubina na esclera e na pele. Geralmente, costuma aparecer, naturalmente, entre o segundo e o terceiro dia de vida e sua duração em média são de dez dias. Conforme estudo, uma quantidade de bilirrubina alta pode levar a encefalopatia e possíveis disfunções neurológicas (NÓBREGA JÚNIOR; VIEIRA; GUEDES JÚNIOR, 2019).

Tabela 4: Apresentação de fatores de risco referente as condições do nascimento das crianças usurárias de CREl's do município de Cuité. Brasil. Paraíba. 2017.

\begin{tabular}{llcc}
\hline \multicolumn{1}{c}{ Variáveis } & $\mathbf{n}$ & $\%$ \\
\hline Idade da criança & & & \\
6 a 11 meses & & 8 & 16,0 \\
\hline
\end{tabular}




\begin{tabular}{lcc}
\hline 1 a 2 anos & 42 & 84,0 \\
Sexo da criança & 26 & 52,0 \\
Feminino & 24 & 48,0 \\
Masculino & 8 & 16,0 \\
Idade gestacional ao nascer & 35 & 70,0 \\
Prematuro & 7 & 14,0 \\
A termo & & \\
Pós termo & 1 & 2,0 \\
Peso aos nascer & 3 & 6,0 \\
$<1000 g$ & 12 & 24,0 \\
$1000-1499 g$ & 34 & 68,0 \\
$1500-2499 g$ & & \\
$\geq 2500 g$ & 8 & 16,0 \\
Apresentou icterícia ao nascer & 42 & 84,0 \\
Sim & 50 & $\mathbf{1 0 0 , 0}$ \\
\hline Não & & \\
Total & & \\
\hline
\end{tabular}

Fonte: Dados da pesquisa, Cuité (2017).

Mesmo diante de constatações acerca das contribuições científicas dos resultados obtidos nesta pesquisa, limitações precisam ser elencadas. Primeiro, no que concerne à abrangência do estudo, que esteve restrito ao município, fragilizando a generalização dos resultados obtidos. Em segundo diz respeito ao número limitado de participantes.

\section{Conclusão}

A partir da realização deste estudo é possível concluir que o desenvolvimento neuropsicomotor infantil é um processo de mudanças complexas e interligadas das quais participam todos os aspectos de crescimento e maturação dos aparelhos e sistemas do organismo.

Para haver um desenvolvimento adequado é necessário que a criança viva em condições favoráveis, e que a exposição de fatores de risco, pré e pósnatais, sejam minimizadas, visto que esses podem comprometer o padrão evolutivo esperado. Constatou-se a presença de fatores de risco importantes na amostra, sendo fundamental a realização de intervenções educativas pautadas nas ações de estimulação e promoção do desenvolvimento infantil a fim de prevenir possíveis alterações.

Assim, consideramos que os objetivos propostos por essa pesquisa trabalho foram cumpridos. No entanto, sugere-se que futuros trabalhos sejam 
desenvolvidos com amostragem maior e que haja uma avaliação do desenvolvimento das crianças para identificação de possíveis alterações conforme suas faixas de idade.

\section{Referências}

ANDRADE, Andréia Moreira de; RAMALHO, Alanderson Alves; KOIFMAN Rosalina Jorge; DOTTO, Leila Maria Geromel; CUNHA, Margarida de Aquino; OPITZ, Simone Perufo. Fatores associados ao uso de medicamentos na gestação em primigestas no Município de Rio Branco, Acre, Brasil. Cad. Saúde Pública, Rio de Janeiro, v. 30, n. 5, p. 1042-56, mai, 2014. Disponível em: http://dx.doi.org/10.1590/0102-311X00172412.

BRASIL, Ministério da Saúde. Manual Aidpi Criança: 2 meses a 5 anos. Brasília, DF, 2017.

BRASIL, Ministério da Saúde. Cadernos de Atenção Básica: atenção ao prénatal de baixo risco. Brasília, DF, 2012.

CAMINHA, Maria de Fátima Costa; SILVA, Suzana Lins da Silva; LIMA, Marília de Carvalho; AZEVEDO, Pedro Tadeu Álvares Costa Caminha de Azevedo; FIGUEIRA, Maria Cristina dos Santos; BATISTA FILHO, Malaquias. Vigilância do desenvolvimento infantil: análise da situação brasileira. Rev Paul Pediatr, v. 35, n. 1, p. 102-09. 2017. Disponível em: http://dx.doi.org/10.1590/19840462/;2017;35;1;00009.

CID, Maria Fernanda Barboza. Cotidiano familiar: refletindo sobre a saúde mental infantil e a prática. Rev Ter. Ocup Univ, São Paulo. V.26, n.3. pp.42838, 2015. Disponível em: https://pt.scribd.com/document/355382975/CotidianoFamiliar-Refletindo-Sobre-a-Saude-Mental-Infantil-e-a-Pratica-de-Atividaes-

Familiares.

CORRÊA, Marianne Dias; TSUNECHIRO, Maria Alice; LIMA, Marlise de Oliveira Pimentel; BONADIO, Isabel Bonadio. Avaliação da assistência prénatal em unidade com estratégia saúde da família. Rev Esc Enferm, v.48 (Espe), p.24-32, 2014. Disponível em: http://www.scielo.br/pdf/reeusp/v48nspe/pt 0080-6234-reeusp-48-esp-024.pdf.

COSTA, Daisy Oliveira; VALENÇA NETO, Paulo da Fonseca; FERREIRA, Luciano Nery; COQUEIRO, Raildo da Silva; CASOTTI, Cezar Augusto. Consumo de álcool e tabaco por gestantes assistidas na estratégia de saúde da família. Revista eletrônica gestão \& saúde. V.05, n. 03, pp.934-48, 2014. Disponível

em:

http://gestaoesaude.unb.br/index.php/gestaoesaude/article/view/572/0.

DAELMANS, Bernadete; BLACK, Maureen; LOMBARDI, Joan; JANE, Lucas; RICHTER, Linda; SILVER, Karlee. Effective interventions and strategies for 
improving early child development. Bmj, v. 351, n. 1, p. 23-6, sept. 2015. Disponível em: https://www.bmj.com/content/bmj/351/bmj.h4029.full.pdf.

EYKEN, Elisa Beatriz Braga Dell'orto Van; GARCIA, Cristiane Sousa Nascimento Baez; ANTUNES, Thuany Medeiros; CAVALCANTE, Ana Beatriz Santana; XARLES, Thiago Moreira; RIBEIRO, Carlos Dimas Matins. Conhecimento sobre desenvolvimento neuropsicomotor da criança. HU Revista, Juiz de Fora, v. 41, n. 1 e 2, p. 23-31, jan./jun. 2015. Disponível em: https://periodicos.ufjf.br/index.php/hurevista/article/view/2466.

FUENTEFRIA, Rubia; SILVEIRA, Rita; PROCIANOY, Renato. Desenvolvimento motor de prematuros avaliados pela Alberta Infant Motor Scale: artigo de revisão sistemática. J. Pediatr, v. 93, n. 4, Jul-Ago. 2017. Disponível em: http://dx.doi.org/10.1016/j.jped.2017.03.003.

GAÍVA, Maria Aparecida Munhoz; MONTESCHIO, Caroline Aparecida Coutinho; MOREIRA, Mayrene Dias de Sousa; SALGE, Ana Karina Marques. Avaliação do crescimento e desenvolvimento infantil na consulta de enfermagem. Av Enferm, v. 36, n. 1, p. 9-21. 2017. Disponível em: http://www.scielo.org.co/pdf/aven/v36n1/0121-4500-aven-36-01-00009.pdf.

GERZON, Laís Rodrigues; CATARINO, Bruna Maciel; Azevedo Kelly Andara de; DEMARCO, Paula Ribeiro; PALMA, Míriam Stock; ALMEIDA, Carla Skilhsn de. Frequência semanal de um programa de intervenção motora para bebês de berçário. Fisioter Pesqui, v. 23, n. 2, p.178-84, 2016. Disponível em: http://www.scielo.br/pdf/fp/v23n2/2316-9117-fp-23-02-00178.pdf.

KIM, Chong; ALBANO, Lilian Maria José; BERTOLA, Débora Romeo. Genética na prática pediátrica. São Paulo: Manole, 2019.

MATOS, Larissa Araujo Matos; CRUZ, Edson Júnior Silva da; CAVALCANTE, Lilia lêda Chaves. Perfil e desenvolvimento neuropsicomotor de crianças beneficiárias e não beneficiárias do Programa Bolsa Família. Mudanças Psicologia da Saúde, v. 25, n. 2, Jul, p. 15-25. 2017. Disponível em: https://www. metodista.br > revistas > index.php > MUD > article > download.

MELO, Neiva Soares de Almeida Melo; MICCIONE, Mariana Morais. As consequências do divórcio dos pais sobre o desenvolvimento infantil: contribuição da abordagem cognitivo-comportamental. Estação Científica, n. 12, julho, p. 1-20. 2014. Disponível em: https://portal.estacio.br/docs\%5Crevista estacao cientifica/03.pdf.

MOREIRA, Rafaela; MAGALHÃES, Lívia; ALVES, Claudia. Efeito do nascimento prematuro no desenvolvimento motor, comportamento e desempenho de crianças em idade escolar: revisão sistemática. J. Pediatr, v .90, n. 2, Mar. 2014. Disponível em: http://dx.doi.org/10.1016/i.jped.2013.05.010.

NAZARETH, Isis Vanessa; SANTOS, Inês Maria Meneses dos Santos; GOLÇALVES, Ana Paula Oliveira; SOUZA, Ester Sena Souza. Risco para o desenvolvimento infantil segundo a estratégia da atenção integrada às doenças 
prevalentes na infância. Rev enferm UFPE on line., Recife, v. 7, n. 2, p. 32836. 2014. DOI: 10.5205/reuol.3073-24791-1-LE.0702201302.

NÓBREGA JÚNIOR, Geraldo de Sousa; VIEIRA, Willian Leite; GUEDES JÚNIOR, José de Arimatea Alves. Icterícia: uma doença comum entre os recém-nascidos. Braz. J. Hea. Rev., v. 2, n. 4, p. 2343-50. 2019. Disponível em: http://www.brjd.com.br/index.php/BJHR/article/view/1696.

PAÍVA, Fernanda da Paz de Souza; SOUZA, Marcela Fernanda da Paz de. Associação entre condições socioeconômicas, sanitárias e de atenção básica e a morbidade hospitalar por doenças de veiculação hídrica no Brasil. Cad. Saúde Pública, v. 34, n. 1, p. 1-11. 2018. Disponível em: https://doi.org/10.1590/0102$\underline{311 \times 00017316 .}$

REICHERT, Altamira Pereira da Silva; COLLET, Neusa; EICKMANN, Sophie Helena; Lima, Marília de Carvalho. Child development surveillance: intervention study with nurses of the Family Health Strategy. Rev. LatinoAm. Enfermagem, v. 23, n. 5, p. 954-62. 2015. Disponível em: http://www.scielo.br/scielo.php?script=sci arttext\&pid=S0104116920150005009 $\underline{54 .}$

RIBEIRO, Débora Gerardo; PEDROSA, Gimol Benzaquen; PADOVANI, Flávia Helena Pereira. Fatores de risco para o desenvolvimento de crianças atendidas em Unidades de Saúde da Família, ao final do primeiro ano de vida. Ciênc. Saúde Coletiva, v. 19, n. 01, Jan. 2014. Disponível em: http://dx.doi.org/10.1590/1413-81232014191.1904.

ROSA, Cristiane Quadrado da; SILVEIRA, Denise Silva da; COSTA, Juvenal Soares Dias da. Fatores associados à não realização de pré-natal em município de grande porte. Rev Saúde Pública, v. 48, n. 6, p. 977-84. 2014. Disponível em:< http://www.scielo.br/pdf/rsp/v48n6/pt 0034-8910-rsp-48-60977.pdf.

SILVA, Thalita Rodrigues; GONTIJO, Cristina Silva. A Família e o Desenvolvimento Infantil sob a Ótica da Gestalt-Terapia. Revista IGT na Rede, v. 13, n. 24, p. 15-36. 2016. Disponível em: http://www.igt.psc.br/ojs.

SILVA, Ângela Cristina Dornelas da; ENGSTRON, Elyne Montenegro; MIRANDA, Cláudio Torres de. Fatores associados ao desenvolvimento neuropsicomotor em crianças de 6-18 meses de vida inseridas em creches públicas do Município de João Pessoa, Paraíba, Brasil. Cad. Saúde Pública, Rio de Janeiro, v. 31, n. 9, p.1881-93, 2015. Disponível em: http://www.scielo.br/scielo.php?pid=S0102311X2015000901881\&script=sci abs tract\&tng=pt.

SILVA, Ângela Cristina Dornelas da; ENGSTRON, Elyne Montenegro; MIRANDA, Cláudio Torres de. Fatores associados ao desenvolvimento neuropsicomotor em crianças de 6-18 meses de vida inseridas em creches públicas do Município de João Pessoa, Paraíba, Brasil. Cad. Saúde Pública, Rio de Janeiro, v. 31, n. 9, p.1881-93, 2015. Disponível em: 
http://www.scielo.br/scielo.php?pid=S0102311X2015000901881\&script=sci abs tract\&tlng=pt.

SOUZA, Juliana Martins; VERÍSSIMO, Maria de La Ó Ramalho. Desenvolvimento infantil: análise de um novo conceito. Rev. Latino-Am. Enfermagem, v. 23, n. 6, p. 1097-104. 2015. Disponível em: http://www.scielo.br/pdf/rlae/v23n6/pt 0104-1169-rlae-23-06-01097.pdf.

SOUZA, Natália dos Santos; PEREIRA, Lilian Paula da Silva; PAULA, Weslla Karla Albuquerque Silva de. Vigilância e estímulo do crescimento e desenvolvimento infantil. Rev enferm UFPE on line, v. 13, n. 3, p. 680-9, 2019. Disponível em: https://doi.org/10.5205/1981-8963-v13i03a238634p680-689$\underline{2019 .}$.

TORQUATO, Isolda Maria Barros; COLLET, Neusa; FORTE, Franklin Delano Soares; FRANÇA, Jael Rúbia Figueiredo de Sá França; SILVA, Maria de Fátima de Oliveira Coutinho; Reichert, Altamira Pereira da Silva. Efetividade de uma intervenção com mães para a estimulação de crianças menores de dois anos. Rev. Latino-Am. Enfermagem, v. 27, n. 3216, p. 1-10. 2019. Disponível em: http://dx.doi.org/10.1590/1518-8345.3176.3216.

ZELKOWITZ, Phyllis. Prematuridade e seu impacto sobre 0 desenvolvimento psicossocial e emocional da criança. Canadá: McGill University, 2017. 